Methodology Prospectively palliative in-patient hospice and community patients had medications deprescribed recorded and the rational. This was prospectively gaining a timeline of medication stops respective to date of death.

Results Data collected from 13/3/18 to 30/06/18 with 647 medication stops of 197 differing medications. 56\% (361) medication stops were due to approaching end-of-life. $15 \%$ (93) due to swallowing difficulties, 17\% (109) due to rationalising medications.

The median number of days of palliative care deprescribing any medication before death is 4 , with $25 \%$ being 1 day and $75 \%$ being 9 days before death. Groups were identified and individually measured, including statins (median 5 days), Betablockers (median 2), ACEi (median 5), anti-platelets (median 9), Warfarin/NOACS (median 5).

Conclusion/discussion By describing deprescribing current practice we outline the short timespan between deprescribing and death for medications arguably of minimal patient benefit at this point in life. The rational for stopping correlates with this postulation, outlining we are stopping the vast majority of medications due to the dying phase rather than preemptively.

\section{PALLIATIVE RADIOTHERAPY IN LUNG CANCER - FUTILE OR WORTHWHILE?}

D Cawley, M Hadaki, A Karathanasi, B Pryke, A McCartney. Wisdom Hospice, Medway NHS Foundation Trust, Maidstone Oncology Centre

\subsection{6/bmispcare-2019-ASP.156}

Background With timely integration of palliative care alongside oncology management, needs of patients are met in a more coherent approach challenging the misperception that there is transition point to palliative care. Therefore the project aimed to understand the timing of radiotherapy in relation to death and inform the clinical decision making regarding the appropriateness of radiotherapy for these patients.

Methods Retrospective case-note review of patient records over a 1 year period (January 2017 to January 2018) who had received radiotherapy with a diagnosis of a lung cancer.

Results 86 patient were identified and the majority had a diagnosis of lung cancer(97\%) with the patients having radiotherapy to their primary lung lesion (35\%), bone metastases (35\%), for malignant spinal cord compression(14\%) and brain metastases(9\%) mainly. $36 \%$ of patients had a performance status (PS) of between $0-1,44 \%$ having a PS of 2 and then 12\% having PS3-4. Interestingly $10 \%$ of patient had no PS recorded. $32 \%$ of patients had one fraction of treatment with $67 \%$ having a fractionated regimen ranging from 3-12 treatments. $16 \%$ of patients died within 30 days having had their treatment and a further $15 \%$ within 59 days with $55 \%$ of those patients having had fractionated regimens (Range 5-12).

Conclusions This project highlights that PS is not always recorded for patient but still a key indicator on the appropriateness of radiotherapy. Given the debate about timing and then effectiveness of radiotherapy and proximity to death, a considerable proportion of patients are having a fractionated regimen before gaining optimal effect of the their treatment. Therefore PS coupled with some understanding of prognosis may help in deciding whether a single high dose fraction maybe more advantageous for the patient than a prolonged fractionation regimen in supporting their symptoms.

\section{COMMUNICATION WITH PALLIATIVE CARE PATIENTS ABOUT DRIVING AND OPIOIDS}

Emma Collins, Andrew Jones. Cwn Taf University Health Board

\subsection{6/bmjspcare-2019-ASP.157}

Background Recent changes in the law regarding liability and driving offences whilst on opioids, highlighted by the MHRA, reinforces the importance of communication about driving whilst taking medication and the law. A robust approach to communicating and documenting this information is required. Methods The audit standard was NICE CG140: 'Opioids in palliative care: safe and effective prescribing of strong opioids for pain in palliative care of adults'. Patients seen in January 2015 in outpatients, day unit, inpatients and the community initiated on opioids or had dose titration, were reviewed. Cases were excluded if had an ECOG of 3-4. The data was analysed and presented at our clinical governance meeting. This triggered a redesign of our inpatient admission proforma. The method of documenting driving status on electronic patient records was changed and promoted. Driving safety leaflets were made available. The audit cycle was completed in June 2017.

Results Pre-implementation, 33 cases were included in analysis. $9 \%$ of cases had their driving status documented. $0 \%$ had documentation of discussion of side effects and $9 \%$ documented that the patient was informed about the consequences of driving on opioids. On re-audit, 20 cases were analysed. Driving status was documented in 50\% of cases, $30 \%$ showed discussion of side effects, and 30\% documented consequences of driving on opioids.

Conclusions It is the clinician's responsibility to appropriately counsel patients on risks of driving whilst taking medications, to provide 'medical defence'. Although conversations often occur, they are seldom documented and can therefore not be evidenced. Having written information to offer can provide evidence, but is no substitute for thorough conversation and eliciting comprehension. In our department, more needs to be done to educate the SPCT to ensure this is done routinely as part of the first assessment, and reviewed frequently, with changes to medications being a prompt.

\section{IS PATIENT-LED FOLLOW-UP (PLFU) AN ACCEPTABLE AND EFFECTIVE SERVICE FOR HOSPICE COMMUNITY PATIENTS? A CROSS SECTIONAL COHORT OF HOSPICE COMMUNITY PATIENTS ON PLFU (LITERATURE SEARCH)}

Rosemary Chester, Stephen Cox. Pilgrims Hospices

\subsection{6/bmjspcare-2019-ASP.158}

Background The current economic climate, increasing cancer survivorship, frailty and palliative care needs has led to a focus on resources. One method being evaluated is patient-led follow-up (PLFU) as a safe, more cost-effective alternative to traditional follow-up. Evidence shows that traditional appointments do not necessarily coincide with patient need or symptoms, and the volume of reviews limits the ability to respond to more urgent needs. Studies in rheumatology and breast cancer patients have demonstrated that nurse-led telephone helplines, education sessions, and open-access in 'low-risk' groups resulted in significant reductions in healthcare utilisation without compromising clinical or psychological wellbeing. 